\title{
Contrast sensitivity and disability glare in the middle years
}

\author{
Joseph M. Harrison and Raymond A. Applegate \\ The University of Texas Health Science Center at San Antonio, San Antonio, Texas 78284 \\ J. Terry Yates \\ School of Aerospace Medicine, Brooks Air Force Base, Texas 78235 \\ Charles Ballentine \\ The University of Texas Health Science Center at San Antonio, San Antonio, Texas 78284
}

Received August 11, 1992; accepted March 2, 1993; revised manuscript received March 3, 1993

\begin{abstract}
Spatial contrast sensitivity and disability glare were measured in a large sample ( $n=90,30$ per decade) of middle-aged subjects, aged 21-50 years, who had clear media and were ophthalmologically normal. We found no significant differences in the contrast sensitivities as a function of age in the middle years for (1) gratings generated on a monitor; (2) interference gratings generated in the retinal plane; (3) gratings in the presence of glare; and (4) mesopic increment thresholds with and without glare. The large sample size provides sufficient statistical power (0.8) for one to conclude that contrast sensitivity, optical quality, and foveal neural sensitivity are unlikely to vary more than $0.1 \mathrm{log}$ unit between the ages of 21 and 50 in ophthalmologically normal subjects with clear media.
\end{abstract}

\section{INTRODUCTION}

Contrast sensitivity (CS) to middle and high spatial frequencies varies with age. ${ }^{1-11}$ The function relating spatial CS to age has not been well defined, particularly in the middle years. Although several studies have shown that CS increases from infancy to a maximum during adolescence $^{1,3,4,11}$ and decreases for older normal subjects (aged 60-80 years), ${ }^{7,9-13}$ sample sizes have not been adequate to determine when the decrease starts. Furthermore, existing studies do not specify the limits of the change that would go undetected in the middle-aged group. That is, it is not clear whether CS decreases continuously from late adolescence or whether there is a critical age after the middle years at which CS begins to decline $e^{5,6,11,14,15}$; nor is it clear in the existing literature how large a change in CS is necessary for detection.

To differentiate between the hypothesis of a continuous CS loss starting in late adolescence and that of a CS loss that begins after the middle years, it is crucial that sample size in each of the decades of the middle years (21-50) be large enough to provide adequate statistical power to detect reasonable effects. Previous studies on CS and aging in' middle-aged subjects have not had sufficient sample sizes to provide acceptable levels of statistical power in the middle years. The lack of sufficient sample size to achieve adequate statistical power is the most likely explanation for the mixed results reported to date. For example, previous research, including ours, suggests a trend toward a decrease in CS in subjects aged 21-40 years. ${ }^{5,8,11,16}$ On the other hand, another study found no statistically significant difference in the CS of subjects aged 19-24 years ( $n=15$ ) compared with that of subjects aged 43-56 years $(n=7) .{ }^{17}$ The number of subjects in the middle years in these studies of CS and aging were not sufficient to test the hypothesis of an age-related change in CS with adequate power. For example, with the use of ten subjects between the ages of 20 and 50 in each decade, the power to detect a moderate effect ${ }^{18,19}(f=0.5$, where $f$ is effect size), at $\alpha=0.05$, where $\alpha$ is a test's significance level, would be unacceptably low (0.37). For the power to reach an acceptably high level (0.8) for the same effect, the same $\alpha$ level, and the same variance, the study would require 30 subjects per decade.

In an effort to resolve the existing ambiguity with respect to the shape of the CS-versus-aging function in the middle years, we conducted the present study (1) to define the CS-aging function in the middle years by using an adequate sample size, and (2) if there is a loss in CS, to determine, by use of measurements of disability glare and neural $\mathrm{CS},{ }^{20-22}$ what portions of the loss can be attributed to optical scatter and/or neural factors.

\section{METHODS}

\section{Subjects}

There were 30 subjects in each decade who ranged in age from 20 to 50 years. The inclusion criteria were as follows:

Medical history No history of eye disease or contact lens use.

Eye exam Must undergo complete eye exam and have

Normal visual acuity (20/20 or better; American Optical Project O Chart), 
Spherical equivalent between -5 and +2 diopters,

Normal confrontation visual fields,

Normal motility (no tropias in the cover test),

Normal anterior chamber (cornea, lens, angle; slit lamp),

Normal fundus, disk macula, and vessels (indirect ophthalmoscope),

Normal intraocular pressure $(<22 \mathrm{~mm} \mathrm{Hg}$; applanation tonometry),

Normal color vision (Farnsworth Panel D15 test).

\section{Apparatus and Procedures}

\section{CS without and with Glare}

The apparatus and procedures have been described in detail in a previous paper. ${ }^{16}$ Briefly, the CS of the right eye was measured monocularly with the head stabilized at $2 \mathrm{~m}$ from the screen of the Tektronix 608 monitor (P31 phosphor) by a chin headrest. Average luminance of the circular 1.5-deg target area of the screen was $68 \mathrm{~cd} / \mathrm{m}^{2}$, and it was surrounded by a white area (16 deg $\times 20 \mathrm{deg})$ set by controlled illumination to $1.7 \mathrm{~cd} / \mathrm{m}^{2}$ without glare and $6.85 \mathrm{~cd} / \mathrm{m}^{2}$ with glare. Contrast of the vertical sinusoidal grating was measured by use of a Pritchard 1980A photometer and was linear from 0 to 0.2 with voltage applied to the $z$ axis of the monitor.

The target area was surrounded by an annular fluorescent-glare source (inner diameter $1.6 \mathrm{deg}$, outer diameter $2.4 \mathrm{deg}$ ). The luminance of the fluorescent lamp was regulated to $16,000 \mathrm{~cd} / \mathrm{m}^{2} \pm 1 \%$ by a photoresistor and was driven at $55 \mathrm{kHz}$, producing 14 lux (lx) at the eye. The illuminance was measured by use of an IL1700 meter with an illuminance head (SED038 1658) with a diffuser (W4218). The fluorescent tube serving as the glare source was set in a keyhole-shaped holder that conformed to the shape of the fluorescent tube. The holder nearly excluded direct spread of the fluorescent light onto the monitor during glare testing. Since it was not possible to exclude all spread of the fluorescent-glare light to the target, it was necessary to establish separate contrast calibration curves depending on whether the glare source was turned on or turned off.

The subjects were instructed and were permitted to practice responding in a simulated paradigm until they thoroughly understood the procedure. Before we determined the five thresholds for each spatial frequency by use of the method of increasing limits, a suprathreshold example of the grating to be detected was presented for $15 \mathrm{~s}$, after which there was a pause of $10 \mathrm{~s}$ plus a randomly variable pause in which the screen remained at zero contrast before it was increased gradually at 0.0014 per $\mathrm{s}$ to a maximum contrast of 0.2 (20\% contrast). The subject was instructed to press a button when the grating became visible. Stimulus presentation and data recording were automated under computer control. The $\log _{10}$ mean CS of the five thresholds was used as the datum defining CS for each spatial frequency. CS's to the four spatial frequencies $[4,12,16$, and 20 cycles per degree (c/deg)] were determined in ascending order with the glare light turned off.
CS's to 4 and $12 \mathrm{c} / \mathrm{deg}$ were determined with the glare light turned on.

\section{Increment Threshold without and with Glare}

The increment-threshold apparatus and method are described in previous papers. ${ }^{23,24}$ Briefly, a centrally viewed 0.5-deg disk flashing at $2 \mathrm{~Hz}$ was adjusted to threshold above a 5-deg square mesopic background $\left(0.4 \mathrm{~cd} / \mathrm{m}^{2}\right)$ in a darkened room. The stimuli were presented in free view with the stimulus-to-eye distance $(0.573 \mathrm{~m})$ fixed by use of a chin headrest. The patient's correction was adjusted appropriately to correct for the working distance. We determined thresholds by use of the method of descending limits. Ten thresholds were determined. The maximum and minimum values were rejected, and the $\log _{10}$ mean of the eight remaining values was defined as the threshold. Glare was produced by a point source (grain-of-rice bulb) $1 \mathrm{deg}$ nasal to the increment stimulus producing $0.22 \mathrm{~lx}$ at the eye. Increment threshold was measured in the same way in the presence of the glare light while the subjects were urged to fixate the increment and to avoid fixating the glare source directly.

\section{CS to Interference Gratings}

CS to interference gratings of 16,20 , and $30 \mathrm{c} / \mathrm{deg}$ were measured by use of a commercial instrument (Randwal LIA). The interference gratings were produced by a $\mathrm{He}-\mathrm{Ne}$ laser $(632.8 \mathrm{~nm})$ and were seen in Maxwellian view as a 2-deg circular target. Alignment was monitored by viewing the pupil through a coaxially aligned television camera. Average luminance of the gratings was equivalent to $7.4 \mathrm{~cd} / \mathrm{m}^{2}$ viewed through a $2.5-\mathrm{mm}$ pupil. The ambient room luminance was $3 \mathrm{~cd} / \mathrm{m}^{2}$. Threshold was determined by use of the method of constant stimuli in the following manner. First, a seed threshold was determined by use of a descending-staircase technique that was repeated until two succeeding staircases resulted in a threshold contrast differing by $<0.5 \log$ unit. During the subsequent full test there were five contrast levels in 0.3log-unit steps that used the already determined seed threshold as the middle of this 1.5-log-unit range. To determine threshold for each spatial frequency tested, we presented 55 trials, with 10 trials for each contrast level and 5 catch trials in random order. A yes-no procedure was used in which the subject indicated detection of the target by pressing a button within $1.5 \mathrm{~s}$ of the offset of the 200 -ms temporally ramped target presentation. The $60 \%$ detection threshold was calculated by use of a linear fit to the probability of detection-log contrast curve.

\section{Testing Sequence}

Testing was conducted in two or three sessions on separate days. The order of the three types of test (CS to monitor, CS to interference gratings, increment threshold) was varied by the experimenter to avoid repetition of the same sequence. Sensitivity without and with glare for CS and increment threshold were measured on the same day. On the few occasions when we measured sensitivity with glare before we measured sensitivity without glare, subjects were permitted to adapt to ambient illumination for 10 min or until they reported the presence of no afterimages resulting from the glare source, whichever was longer. 


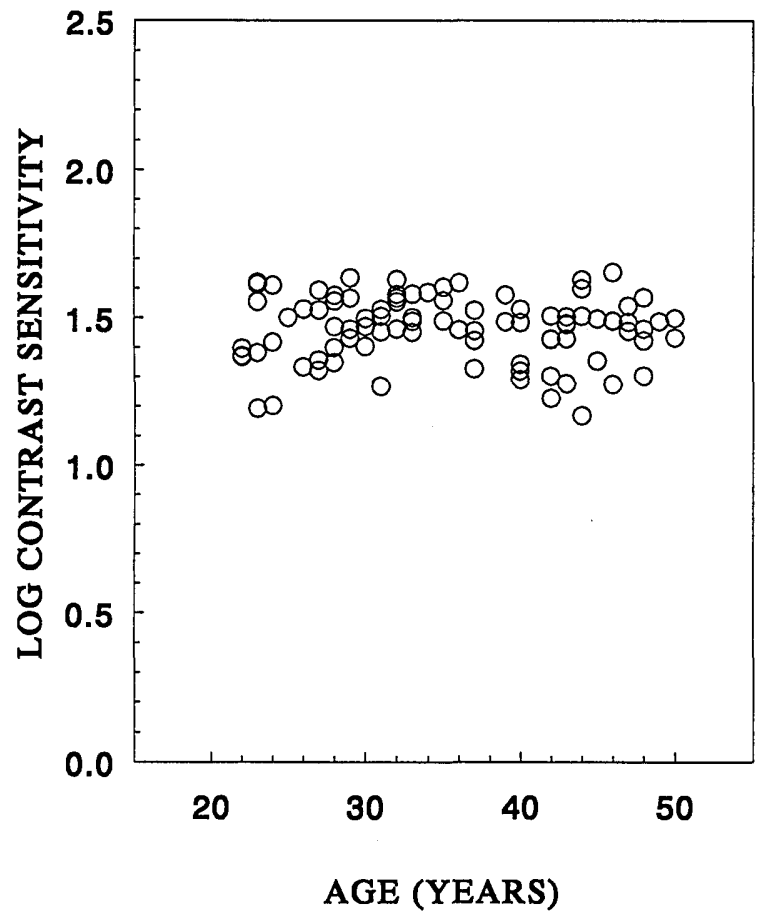

Fig. 1. $\log _{10}$ contrast sensitivity to $16 \mathrm{c} / \mathrm{deg}$ [cathode-ray-tube (CRT) presentation] for each of the 90 subjects as a function of age.

\section{RESULTS}

\section{CS without Glare}

Figure 1 graphically displays the log CS without glare at $16 \mathrm{c} / \mathrm{deg}$ for each subject. These data are representative of the variability and trend of the data for all spatial frequencies. Consistent with the visual impression, statistical analysis reveals that CS remains constant during the middle years $\left[r^{2}=0.0012\right.$, slope $=-4.6 \times 10^{-4}$ not significantly different from zero $(p=0.78)]$.

Figure 2 shows the mean $\log$ CS for $4,12,16$, and $20 \mathrm{c} / \mathrm{deg}$ for the three age groups. Two-way analysis of variance of the $\log$ CS data with repeated measures on one factor, spatial frequency (STATISTICA by StatSoft), showed that age group and age group $\times$ spatial-frequency interaction were not significant factors $(p=0.27$ and 0.62 , respectively). As anticipated, CS varied with spatial frequency for all age groups ( $p \ll 0.001)$.

\section{CS without and with Glare}

Mean $\log$ CS for glare and no-glare conditions for the three age groups are plotted in Fig. 3. CS is consistently greater without than with glare, and there are no obvious differences in the glare effect across the age groups for either 4 or $12 \mathrm{c} / \mathrm{deg}$. There was a slightly greater effect of glare on CS to $12 \mathrm{c} / \mathrm{deg}$ than on CS to $4 \mathrm{c} / \mathrm{deg}$. A statistical analysis with the use of three factors and repeated measures on spatial frequency and no-glare/glare factors revealed the following: (1) The difference in CS without and with glare was significant $(p \ll 0.001)$. (2) Glare caused a significant decrease of CS of $\sim 0.12 \mathrm{log}$. (3) The interaction between age and glare conditions was not significant $(p=0.36)$. (4) The interactions between age and spatial frequency and among age, glare condition, and spatial frequency were also not significant. (5) However, the interaction between spatial frequency and glare condition was significant ( $p=0.027$ ), confirming the impression of a slightly greater effect of glare on 12 than on $4 \mathrm{c} / \mathrm{deg}$.

The power of the negative tests was relatively high (0.8) to detect a moderate effect $(f=0.5)$. An effect of this magnitude for these data would correspond to just under $0.1 \log$ CS. Therefore, if there is a significant age-related change of CS or glare susceptibility in the 21-50-year age range, the effect is likely to be smaller than $0.1 \log$ CS.

\section{Increment Threshold without and with Glare}

In Figs. 4 and 5, the increment threshold data are plotted as $\log I / \Delta I$ rather than the conventional $\log \Delta I / I$ to facilitate comparisons with CS, for which increased sensitivity is indicated by larger values. No dominant age trend of $\log I / \Delta I$ measured during glare is indicated by the data in Fig. 4.

Visual inspection of Fig. 5 shows that there is a large effect of glare on mean log $I / \Delta I$, that the effect was not different for the three age groups, and that there is no age trend for data measured during either the no-glare or the glare condition.

Statistical analysis (two-factor analysis of variance with repeated measures on glare condition) confirmed that the effect of age and the interaction between age and glare condition were not significant $(p=0.62$ and $p=0.36$, respectively) and that the effect of glare was significant $(p \ll 0.001)$.

\section{CS to Interference Gratings}

The false-alarm rate was very low, and the linear fits to the individual psychometric curves had high correlation coefficients ( $r=0.7$ or greater). Log CS for a $16-\mathrm{c} / \mathrm{deg}$ grating is shown for each subject in Fig. 6. Again, there

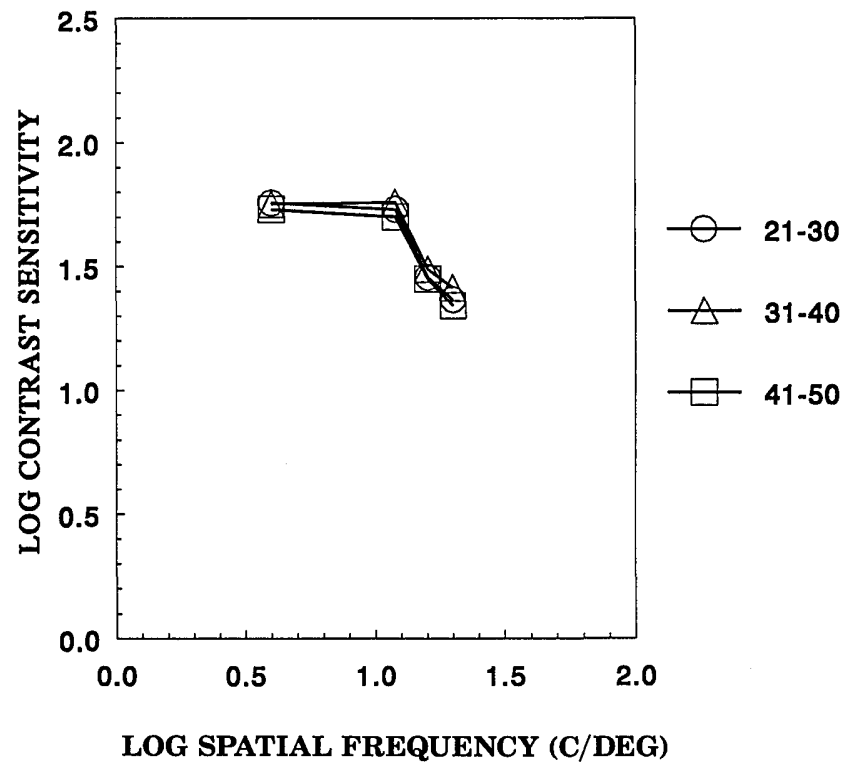

Fig. 2. Mean $\log _{10}$ contrast sensitivity (CRT presentation) as a function of $\log _{10}$ spatial frequency for each of the three age groups ( $n=30$ for each group). One standard error of the mean was less than the size represented by the symbols. The lines connect the points and are not fitted curves. 
is no indication of a trend with age $\left[r^{2}=0.02\right.$, slope $=$ $-2.57 \times 10^{-3}$ not significantly different from zero $(p=$ $0.17)$, and the data show scatter that is similar to that of the CRT data despite the fact that we used different presentation and psychophysical techniques. Consistent with previous results, ${ }^{21,22,25}$ the CS to interference gratings is larger than that to gratings of the same spatial frequency presented on a CRT.

Mean $\log$ CS as a function of spatial frequency of interference gratings for the three age groups can be seen in Fig. 7. As with the ocularly imaged gratings, there is almost complete overlap of the data. Log CS to interfer ence gratings did not vary significantly with age ( $p=$ 0.91 ). The interaction between age and spatial frequency was not significant ( $p=0.81$ ), and again, as anticipated, CS varied significantly with spatial frequency $(p \ll$ 0.001 ). Therefore CS varied with spatial frequency in the same way for all age groups.

\section{DISCUSSION}

We were unable to demonstrate a significant CS-age relation in a middle-aged population. Therefore our large-sample data strongly indicate that the loss of CS ex-

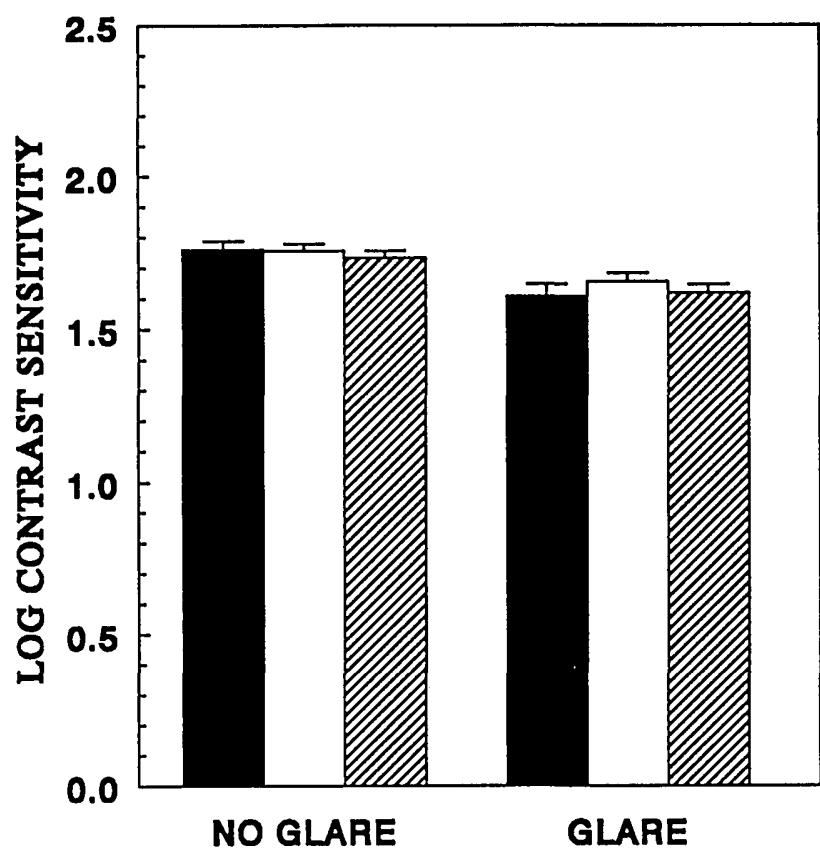

$21-30$
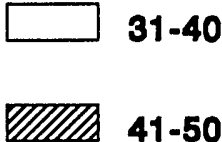

41-50

(a)
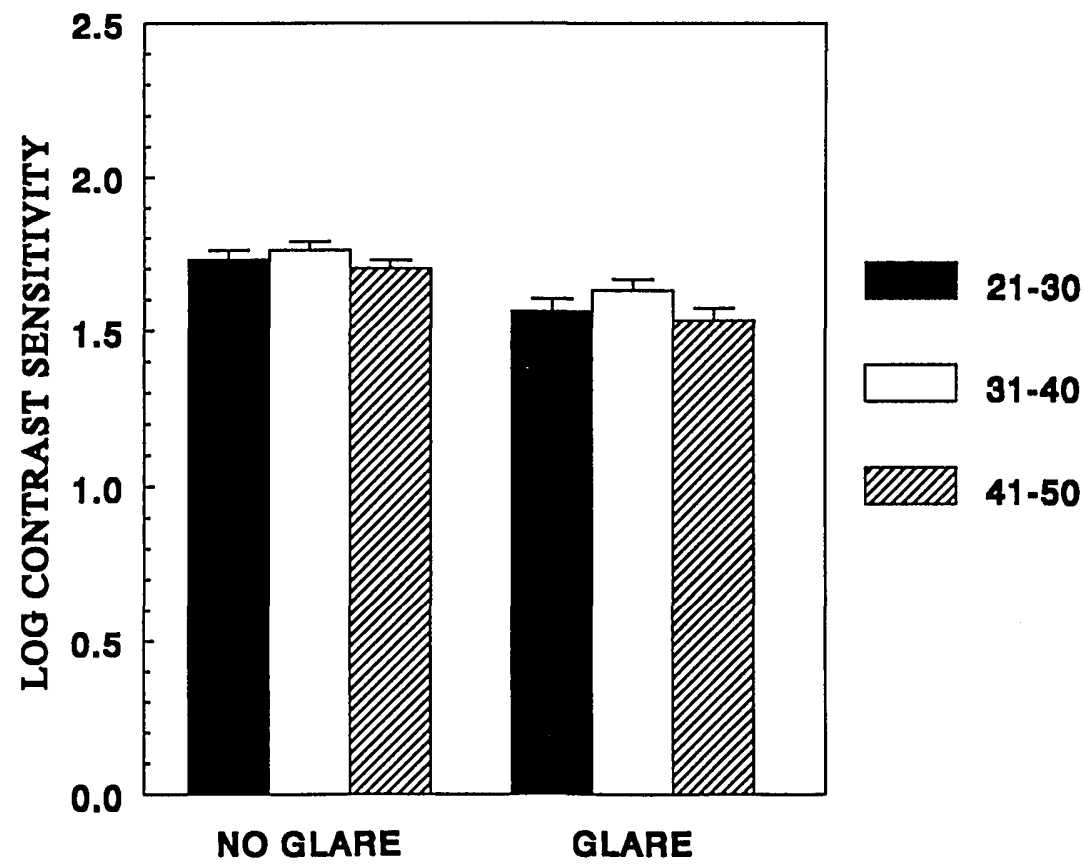

(b)

Fig. 3. Mean $\log _{10}$ contrast sensitivity for each age group without glare and with glare for (a) 4-c/deg and (b) 12-c/deg CRT. 


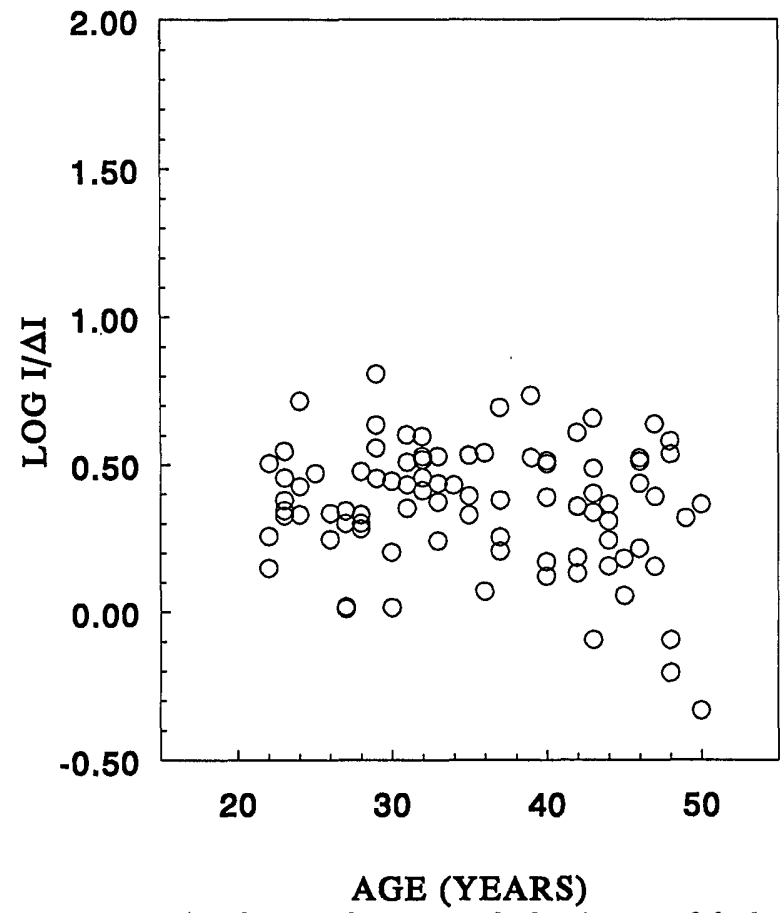

Fig. 4. $\log _{10} I / \Delta I$ glare condition (i.e., the luminance of the background divided by the luminance of the flashing test stimulus) for each of the 90 subjects as a function of age.

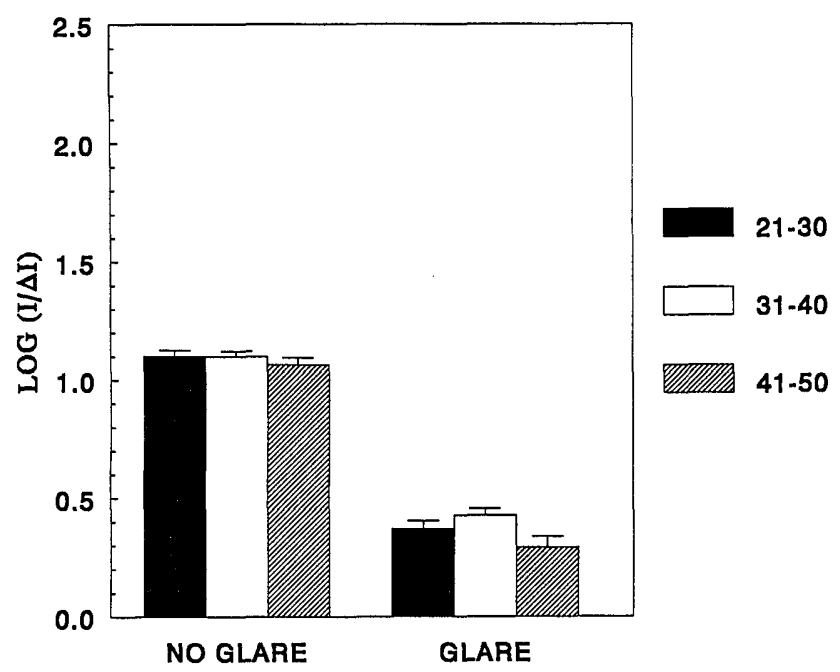

Fig. 5. Small-spot increment threshold: mean $\log _{10} I / \Delta I$ for each age group without and with glare. One standard error of the mean is plotted above each bar.

perienced by older subjects must be occurring after the age of 50. This is consistent with the Johnson-Choy ${ }^{14}$ analysis of visual function and aging. However, it is not consistent with the conclusions of Owsley et al. ${ }^{15}$ who argued that a bilinear model was not a significant improvement over a linear model to describe the changes of CS with age. The superiority of the linear model implies a continuous visual-aging process after the age of 20 . Like Owsley et al.., ${ }^{15}$ Morrison and McGrath ${ }^{6}$ found that there was a linear decrease in CS to interference gratings. However, they also report that the CS to gratings presented on a CRT was relatively invariant in the comparatively small sample of 21-50-year-olds and decreased after the age of 50. In another study CS to CRT gratings was found not to vary significantly for two groups of subjects aged 19-24 and 43-56 years. ${ }^{17}$ The number of subjects in these studies, however, was not sufficient to provide adequate power to place reasonable limits on effect sizes that could be detected.

One might expect that glare paradigms would enhance any small effect of age resulting from light scatter so that the age effect on CS with glare would be more apparent than for CS to gratings or increment threshold without

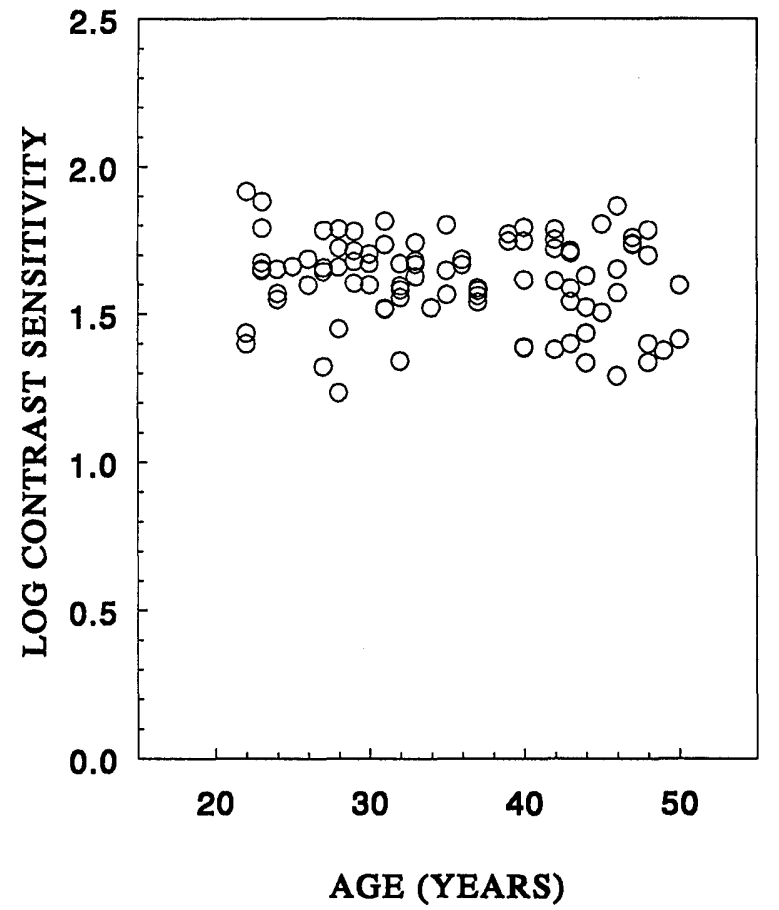

Fig. 6. $\log _{10}$ contrast sensitivity (interference-grating presentation) to 16-c/deg gratings for each of the 90 subjects as a function of age.

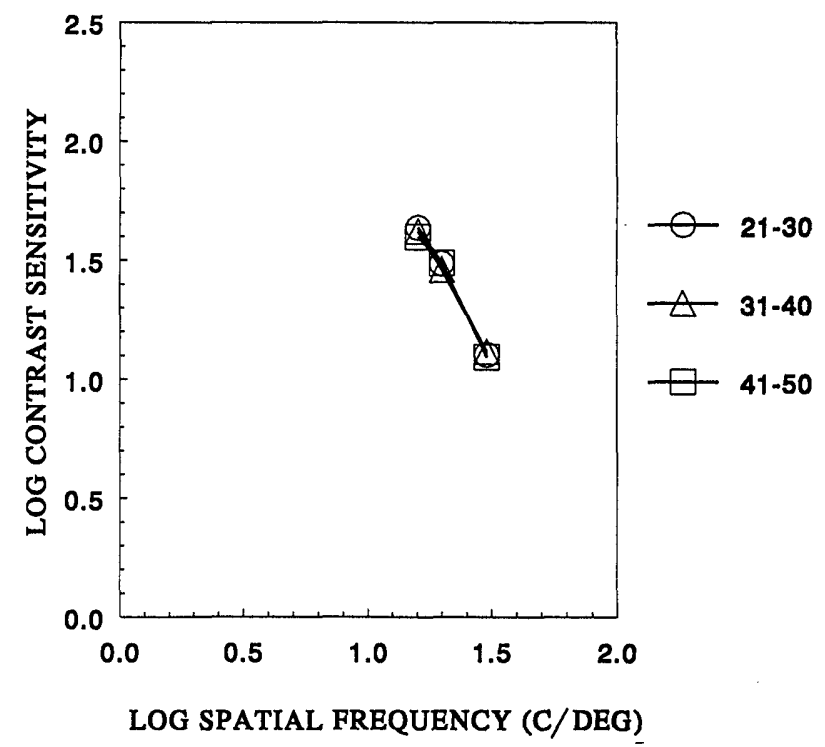

Fig. 7. Mean $\log _{10}$ contrast sensitivity (interference-grating presentation) as a function of $\log _{10}$ spatial frequency for each of the three age groups. The lines connect the points and are not fitted to the data. 
glare. ${ }^{24,26-28}$ We found no evidence of a larger change of CS with age during glare in this study. We found that glare susceptibility does not change significantly with age in a large group of ophthalmologically normal middle-aged subjects. This finding is consistent with the results of other studies ${ }^{6,12,17,29,30}$ and indicates that there were no subclinical age-related increases of light scatter detectable by our tests in this age group. This conclusion is also consistent with the trend of the data in the study of IJspeert et $a l .{ }^{31}$ who used a more direct compensation method to assess forward ocular light scatter. However, one study ${ }^{32}$ found a significant change in disability glare (incrementthreshold technique) between the age groups of 20-25 $(n=10)$ and $40-45(n=10)$ years. The $40-45$-year-old group had $0.08 \log \Delta I$ more elevation of increment threshold during glare than did the 20-25-year-old group. Interestingly, there was no significant difference between the disability glare of the 60-65- $(n=10)$ and 40-45-yearold groups, which suggests that the significant result reflected sampling error.

In the five increment-threshold studies of disability glare and age reviewed by Vos, ${ }^{33}$ the predicted change in $\Delta I$ ranged from $0.06 \log$ to $0.21 \mathrm{log}$, depending on the study, from age 25 to age 45 . Our study had a power of 0.8 to detect a 0.05 -log-unit change at the 0.05 level of confidence for $\log I / \Delta I$. Therefore the lowest of the age factors would be more consistent with our results. In his expression to predict equivalent luminance, $\operatorname{Vos}^{33}$ used the second-to-lowest value that would predict a change of $0.07 \log \Delta I$ from age 25 to age 45 . If there are age-related changes in disability glare assessed by increment threshold from age 21 to age 50, they must be quite small.

In conclusion, in this study we found no support for statistically significant age-related changes in CS or light scatter assessed by disability glare in visually normal subjects aged 21-50 years. Any such age-related changes are statistically likely to be $<0.1 \log$ CS for gratings and $0.05 \log \Delta I$ for increment threshold. The stability of CS with age during glare and no glare in this age group, coupled with the demonstrated significant differences in CS between ophthalmologically normal young and old subjects, ${ }^{12}$ implies that the function relating CS and disability glare to age becomes steeper after the age of 50 .

\section{ACKNOWLEDGMENTS}

This research was supported in part by U.S. Air Force contract C-F33615-87-D-0609 and by an unrestricted development grant from Research to Prevent Blindness, Inc., to the Department of Ophthalmology at the University of Texas Health Science Center at San Antonio. R. Applegate's research was supported by National Institutes of Health grant EY 08520.

\section{REFERENCES}

1. K. Arundale, "An investigation into the variation of human contrast sensitivity with age and ocular pathology," Br. J. Ophthalmol. 62, 213-215 (1978).

2. G. Derefeldt, G. Lennerstrand, and B. Lundh, "Age variations in normal human contrast sensitivity," Acta Ophthalmol. 57, 679-690 (1979).

3. L. D. Beazley, D. J. Illingworth, A. Jahn, and D. V. Greer, "Contrast sensitivity in children and adults," Br. J. Ophthalmol. 64, 863-866 (1980).
4. A. Bradley and R. D. Freeman, "Contrast sensitivity in children," Vision Res. 22, 953-959 (1982).

5. C. Owsley, R. Sekuler, and D. Siemsen, "Contrast sensitivity throughout adulthood," Vision Res. 23, 689-699 (1983).

6. J. D. Morrison and C. McGrath, "Assessment of the optical contributions to the age-related deterioration in vision," Q. J. Exp. Physiol. 70, 249-269 (1985).

7. J. E. Ross, D. D. Clarke, and A. J. Bron, "Effect of age on contrast sensitivity function: uniocular and binocular findings," Br. J. Ophthalmol. 69, 51-56 (1985).

8. K. E. Higgins, M. J. Jaffe, R. C. Caruso, and F. M. deMonasterio, "Spatial contrast sensitivity: effects of age, test-retest, and psychophysical method," J. Opt. Soc. Am. A 5, 2173-2180 (1988).

9. M. E. Sloane, C. Owsley, and S. L. Alvarez, "Aging, senile miosis and spatial contrast sensitivity at low luminance," Vision Res. 28, 1235-1246 (1988).

10. M. E. Sloane, C. Owsley, and C. A. Jackson, "Aging and luminance-adaptation effects on spatial contrast sensitivity," J. Opt. Soc. Am. A 5, 2181-2190 (1988).

11. U. Tulunay-Keesey, J. N. Ver Hoeve, and C. Terkla-McGrane, "Threshold and suprathreshold spatiotemporal response throughout adulthood," J. Opt. Soc. Am. A 5, 2191-2200 (1988).

12. D. B. Elliott, "Contrast sensitivity decline with ageing: a neural or optical phenomenon?" Ophthalmic Physiol. Opt. 7, 415-419 (1987).

13. D. B. Elliott, D. Whitaker, and D. MacVeigh, "Neural contribution to spatiotemporal contrast sensitivity decline in healthy ageing eyes," Vision Res. 30, 541-547 (1990).

14. M. A. Johnson and D. Choy, "On the definition of age-related norms for visual function testing," Appl. Opt. 26, 1449-1454 (1987).

15. C. Owsley, K. Knoblauch, and C. Katholi, "When does visual aging begin?" Invest. Ophthalmol. Vis. Sci. Suppl. 33, 1414 (1992).

16. J. T. Yates, J. M. Harrison, P. S. O'Connor, and C. Ballentine, "Contrast sensitivity: characteristics of a large, young, adult population," Am. J. Optom. Physiol. Opt. 64, 519-527 (1987).

17. D. Finlay and J. Wilkinson, "The effects of glare on the contrast sensitivity function," Hum. Factors 26, 283-287 (1984).

18. J. Cohen, Statistical Power Analysis for the Behavioral Sciences, 2nd ed. (Erlbaum, Hillsdale, N.J., 1988).

19. E. C. Susskind and E. W. Howland, "Measuring effect magnitude in repeated measures ANOVA designs: implications for gerontological research," J. Gerontol. 35, 867-876 (1980).

20. D. G. Green, "Testing the vision of cataract patients by means of laser-generated interference fringes," Science 168, 12401242 (1970).

21. M. S. Banks, W. S. Geisler, and P. J. Bennett, "The physical limits of grating visibility," Vision Res. 27, 1915-1924 (1987).

22. E. R. Jankelovits, S. J. Lichtenstein, S. Groll, P. W. Remijan, and J. Hirsch, "Assessment of retinal function in cataract patients by a combination of laser interferometry and conventional display methods to measure contrast sensitivity," Appl. Opt. 27, 1057-1063 (1988).

23. J. M. Harrison, C. Peng, and C. Ballentine, "Glare and age: acquisition of a database for aircrew standards," U.S. Air Force SAM-TR-90-28 (U.S. Government Printing Office, Washington, D.C., 1990).

24. R. A. Applegate, L. R. Trick, D. L. Meade, and J. Hartstein, "Radial keratotomy increases the effects of disability glare: initial results," Ann. Ophthalmol. 19, 293-297 (1987).

25. F. W. Campbell and D. G. Green, "Optical and retinal factors affecting visual resolution," J. Physiol. (London) 18, 576-593 (1965).

26. R. F. Hess and L. F. Garner, "The effect of corneal edema on visual function," Invest. Ophthalmol. Vis. Sci. 16, 5-13 (1977).

27. L. G. Carney and R. J. Jacobs, "Mechanisms of visual loss in corneal edema," Arch. Ophthalmol. 102, 1068-1071 (1984).

28. L. E. Paulsson and J. Sjostrand, "Contrast sensitivity in the presence of a glare light. Theoretical concepts and preliminary clinical studies," Invest. Ophthalmol. Vis. Sci. 19, 401406 (1980).

29. G. Verriest and A. Uvijls, "Disability glare in normal and diseased eyes," Clin. Vision Sci. 4, 253-256 (1989). 
30. D. Yager, T. Hage, R. Yuan, S. Mathews, and M. Katz, "The relations between contrast threshold, lens back scatter, and disability glare," in Noninvasive Assessment of the Visual System, Vol. 3 of 1990 OSA Technical Digest Series (Optical Society of America, Washington, D.C., 1990), pp. 168-171.

31. J. K. IJspeert, P. W. de Waard, T. J. van den Berg, and P. T. de Jong, "The intraocular straylight function in 129 healthy volunteers; dependence on angle, age and pigmentation," Vision Res. 30, 699-707 (1990).
32. S. P. Sturgis and D. J. Osgood, "Effects of glare and background luminance on visual acuity and contrast sensitivity: implications for driver night vision testing," Hum. Factors 24, 347-360 (1982).

33. J. J. Vos, "Disability glare-a state of the art report," CIE J. 3, 39-53 (1984). 